\title{
Local signatures of electron-electron scattering in an electronic cavity
}

\author{
Carolin Gold $\odot,{ }^{1, *}$ Beat A. Bräm $\odot,{ }^{1}$ Richard Steinacher, ${ }^{1}$ Tobias Krähenmann $\odot,{ }^{1}$ Andrea Hofmann, ${ }^{1}$ Christian Reichl, ${ }^{1}$ \\ Werner Wegscheider $\odot,{ }^{1}$ Mansour Shayegan $\odot,{ }^{2}$ Klaus Ensslin $\odot,{ }^{1}$ and Thomas $\operatorname{Ihn}^{1}$ \\ ${ }^{1}$ Laboratory for Solid State Physics, ETH Zurich, 8093 Zurich, Switzerland \\ ${ }^{2}$ Department of Electrical Engineering, Princeton University, Princeton, New Jersey 08544, USA
}

(Received 14 January 2021; revised 11 February 2021; accepted 12 February 2021; published 29 March 2021)

\begin{abstract}
We image equilibrium and nonequilibrium transport through a two-dimensional electronic cavity using scanning gate microscopy. Injecting electrons into the cavity through a quantum point contact close to equilibrium, we raster scan a weakly invasive tip above the cavity region and measure the modulated conductance through the cavity. Varying the electron injection energy between $\pm 2 \mathrm{meV}$, we observe that conductance minima turn into maxima beyond an energy threshold of $\pm 0.6 \mathrm{meV}$. This observation bears similarity to previous measurements by Jura et al. [Phys. Rev. B 82, 155328 (2010)], who used a strongly invasive tip potential to study electron injection into an open two-dimensional electron gas. This resemblance suggests a similar microscopic origin based on electron-electron interactions.
\end{abstract}

DOI: 10.1103/PhysRevResearch.3.013287

\section{INTRODUCTION}

Electron-electron (e-e) interactions and their role in electron transport are a topic of continuing interest in mesoscopic physics. Thanks to momentum conservation in e-e scattering processes, the latter do not influence the electron mobility unless paired with another scattering mechanism [1]. Interactions have been found to impact, e.g., the conductivity of disordered systems via Friedel oscillations around screened impurities [2,3], and are the key low-temperature decoherence mechanism in quantum transport experiments [4] such as the Aharonov-Bohm effect [5-7] or weak localization [8]. Recently, renewed interest has arisen in viscous effects observed in electron liquids at elevated temperatures $[9,10]$.

The rich variety of existing experiments includes attempts to probe e-e scattering by injecting nonequilibrium electrons into an equilibrium Fermi sea $[11,12]$. Among them is the work by Jura et al. [13], which inspired the experiments we present here. In Ref. [13], the flow of electrons injected through a quantum point contact is imaged at energies above the thermal smearing of the Fermi-Dirac distribution. Raster scanning a locally depleting scanning gate tip above the open electron gas downstream of the injection point, the authors observed a contrast inversion of the branched electron flow signal at elevated source-drain bias voltages. They interpreted this contrast inversion as a manifestation of e-e scattering in the electron gas.

\footnotetext{
*cgold@phys.ethz.ch

Published by the American Physical Society under the terms of the Creative Commons Attribution 4.0 International license. Further distribution of this work must maintain attribution to the author(s) and the published article's title, journal citation, and DOI.
}

Our experiments aim at exploring this effect for the socalled weakly invasive tip potentials induced by the scanning gate. In general, most scanning gate experiments (including branched electron flow measurements behind a point contact [14-16]) require a tip-induced potential which depletes the electron gas locally (strongly invasive regime). However, we recently found a method to significantly enhance the sensitivity at nondepleting voltages (weakly invasive regime) [17], thus reducing the influence of the tip on the unperturbed system. This method utilizes a gate defined, open cavity structure [18-20], which concentrates the scattering density of states behind the quantum point contact and thereby enables scanning gate experiments at strongly reduced voltages applied to the scanning gate. In this paper, we operate such a structure in the nonlinear bias regime and, in this modified setting, find the interaction effects previously observed for electron injection into an open two-dimensional electron gas. Our finding may help unravel the microscopic details of this effect by theoretical means beyond the explanation given in Ref. [13].

\section{SAMPLE AND EXPERIMENTAL SETUP}

Our measurements are performed on the open resonator structure depicted in Fig. 1(a) at temperature $T=270 \mathrm{mK}$. The sample is based on a $\mathrm{Ga}(\mathrm{Al}) \mathrm{As}$ heterostructure [dark grey in Fig. 1(a)] in which a two-dimensional electron gas (2DEG) with electron density $n=1.9 \times 10^{11} \mathrm{~cm}^{-2}$ and mobility $\mu=$ $4.4 \times 10^{6} \mathrm{~cm}^{2} / \mathrm{Vs}$ is formed $90 \mathrm{~nm}$ below the surface. Negative gate voltages, applied to the $300 \mathrm{~nm}$ wide quantum point contact (QPC) and arc-shaped cavity gate [light grey in Fig. 1(a)], form a $2 \mu \mathrm{m}$ long resonator with an opening angle of $90^{\circ}$ centered around the QPC.

Applying a bias voltage $V_{\mathrm{sd}}=V_{\mathrm{sd}, \mathrm{ac}}+V_{\mathrm{sd}, \mathrm{dc}}$ between the source (S) and grounded drain (D) contacts, we perform both equilibrium and nonequilibrium measurements of the 
(a)

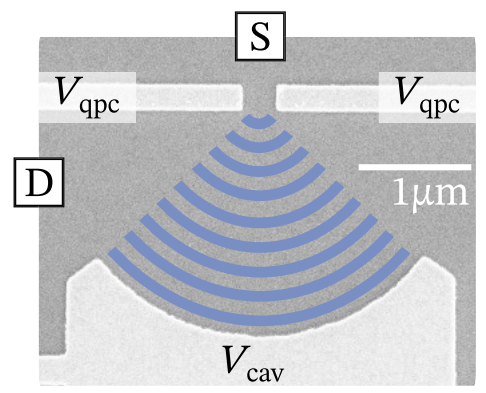

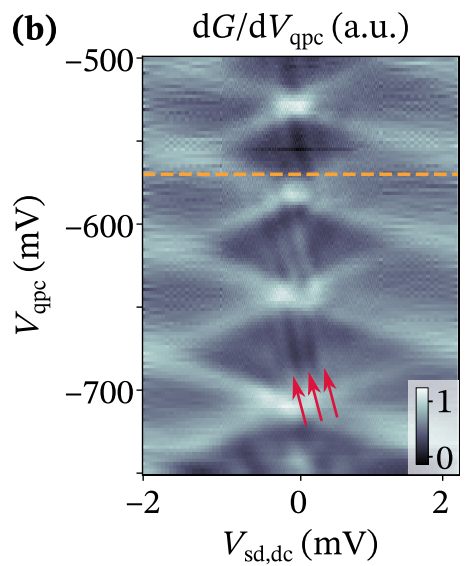

(c)

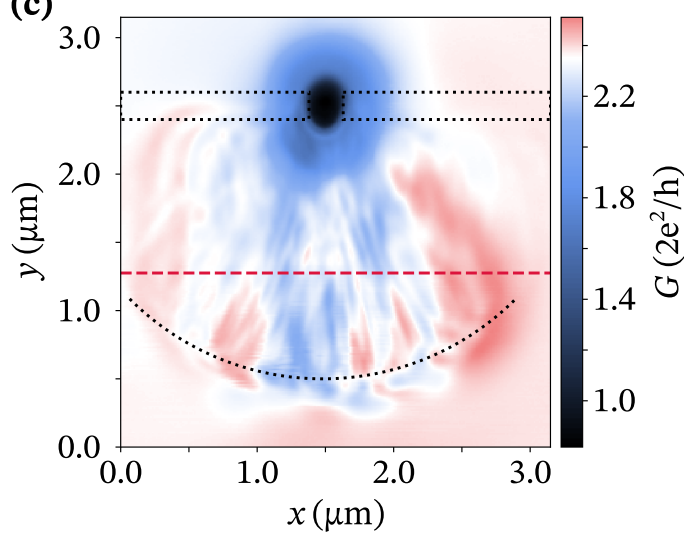

FIG. 1. (a) Scanning electron micrograph of the open resonator structure with Schottky gates (light gray) on a GaAs-surface (dark gray). The cavity area is depicted by the blue standing wave. The black squares denote the source (S) and drain (D) ohmic contacts. This micrograph has also been published in Ref. [17] and the Supplementary Materials of Ref. [21]. (b) Numerical derivative $d G / d V_{\mathrm{qpc}}$ of the differential conductance $G$ as a function of $V_{\mathrm{sd}, \mathrm{dc}}$ and $V_{\mathrm{qpc}}$. Red arrows mark the cavity modes. The dashed orange line corresponds to the QPC voltage used for all following measurements. The data for $\left|V_{\mathrm{sd}, \mathrm{dc}}\right|>1 \mathrm{mV}$ are obtained with lower resolution in bias voltage and are numerically smoothened to increase the visibility of the diamond-shaped pattern. An excerpt from this data has also been published in the Supplementary Material of Ref. [21]. (c) Spatial image of the conductance $G(x, y)$ in the cavity as a function of the tip position for $V_{\text {tip }}=-1 \mathrm{~V}$ and $V_{\text {cav }}=-400 \mathrm{mV}$. Dotted lines outline the position of the Schottky gates. The data presented in Fig. 2 are obtained along the red dashed line.

differential conductance $G=I_{\text {sd, ac }} / V_{\text {sd,ac }}$ through the sample. Here, $I_{\mathrm{sd}, \mathrm{ac}}$ is the measured source-drain current, $V_{\mathrm{sd}, \mathrm{ac}}=$ $50 \mu \mathrm{V}_{\mathrm{rms}}$ for all measurements, and the dc voltage is varied between $V_{\text {sd,dc }} \in[-2 \mathrm{mV}, 2 \mathrm{mV}]$.

To explore the local properties of electron transport through the open resonator, we perform scanning gate microscopy (SGM) measurements. To this end, we raster scan a voltage-biased metallic tip approximately $65 \mathrm{~nm}$ above the open resonator structure while measuring the differential conductance $G(x, y)$ as a function of the tip position $(x, y)$. Unless stated otherwise, the tip is biased at a voltage $V_{\text {tip }}=-1 \mathrm{~V}$, which induces a tip potential with an amplitude much smaller than the Fermi energy $E_{\mathrm{F}}$ [17]. Electrons interacting with this weakly invasive, tip-induced potential are not backscattered by a hard-wall potential [14,15,22], but rather experience gentle electron deflection.

\section{CHARACTERIZATION OF THE CAVITY}

\section{A. Characterization of the cavity in absence of the SGM tip}

We first characterize the open resonator in absence of the tip by measuring the differential conductance $G\left(V_{\mathrm{sd}, \mathrm{dc}}, V_{\mathrm{qpc}}\right)$. The numerical derivative $d G / d V_{\mathrm{qpc}}$ of the latter is depicted in Fig. 1(b) and exhibits the diamond pattern characteristic for nonequilibrium electron transport through QPCs. The dark rhombi with $d G / d V_{\mathrm{qpc}} \approx 0$ correspond to regions with constant differential conductance on a conductance plateau, their extent in bias direction yielding the subband spacing $\Delta_{\mathrm{sb}}=$ $1.5 \mathrm{meV}$. All the following measurements are performed at $V_{\mathrm{qpc}}=-570 \mathrm{mV}$ [cf. the orange dashed line in Fig. 1(b)], for which the QPC conductance is tuned to the third conductance plateau in absence of the cavity. In addition to the diamondshaped pattern, we observe parallel and equally spaced lines in the differential conductance in the region of the QPC plateaus [cf. the red arrows in Fig. 1(b)]. These lines are observed for nonzero cavity gate voltages only and are a manifestation of cavity modes with an average energy spacing $\Delta E_{\text {cav }}=$ $236 \pm(19) \mu \mathrm{eV}[23,24]$.

\section{B. Characterization of the cavity in presence of the SGM tip}

To study the conductance through the cavity on a local scale, we raster scan the SGM tip above the whole resonator area formed between the QPC and cavity gate. The differential conductance $G(x, y)$ measured at different tip positions $(x, y)$ within this area is depicted in Fig. 1(c). In agreement with previous work on the same sample [17], it exhibits a distinct spatial structure of fine conductance modulations which emanate from the QPC radially. These conductance modulations arise from the influence of the tip-induced potential on the local density of scattering states, which emanate from the QPC into the cavity and are concentrated in the latter [21].

The average conductance $G(x, y)$ in Fig. 1(c) is reduced with respect to the conductance of $G=3 \times 2 e^{2} / h$ on the third QPC plateau due to the capacitive action of the cavity gate and the tip on the QPC channel (see Appendix A for further details). The observed asymmetry of the conductance modulations with respect to the mirror axis of the cavity $(x \approx 1.5 \mu \mathrm{m})$ originates from slight asymmetries in the cavity design or the background potential. The latter induce an asymmetry in the local density of scattering states in the cavity which results in the observed asymmetry in the tip-induced conductance modulations [cf. Fig. 1(c)]. While this asymmetry is important for the exact tip position at which a minimum (maximum) occurs, we do not expect it to influence the observation of the minima-to-maxima transition discussed in the following.

\section{FINITE BIAS MEASUREMENTS}

A. Finite bias measurements in presence of the SGM tip

Based on previous measurements on an open 2DEG behind a QPC [13], we perform SGM measurements at finite 

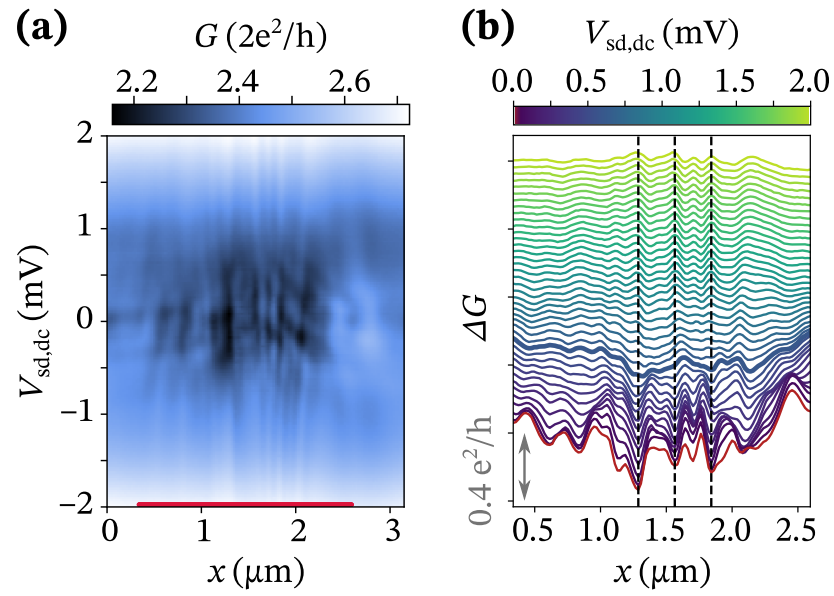

FIG. 2. Differential conductance $G\left(x, V_{\mathrm{sd}, \mathrm{dc}}\right)$ along the dashed red line in Fig. 1(c). (a) Raw data. (b) Linewise conductance difference $\Delta G(x)$ as a function of the tip position $x$ along the red line in (a) for small steps in $V_{\text {sd,dc }}$. The red curve denotes the conductance at $V_{\text {sd,dc }}=0 \mathrm{mV}$ and the lines are offset with respect to each other. Black dashed guides to the eye denote three exemplary minimum-to-maximum transitions. The thick line marks the conductance modulations at the transition voltage.

source-drain voltages in order to probe e-e scattering in the open resonator on a local scale. The measured differential conductance $G\left(x, V_{\mathrm{sd}, \mathrm{dc}}\right)$ for tip positions $x$ along the dashed red line in Fig 1(c) and bias voltages $V_{\mathrm{sd}, \mathrm{dc}}$ is depicted in Fig. 2(a).

At zero source-drain bias we recover the conductance modulations $G \in[2.15,2.5] \cdot 2 e^{2} / h$ already observed along the dashed red line in the spatial cavity map [cf. Fig. 1(c)]. With increasing source-drain bias $V_{\text {sd,dc }}$, the overall conductance $G$ through the sample increases while maintaining its distinct spatial modulation. Minima (or maxima) of the conductance occur at exactly the same tip positions $x$ for source-drain biases of up to approximately $0.6 \mathrm{mV}$. However, this changes significantly for bias voltages $\left|V_{\mathrm{sd}, \mathrm{dc}}\right|>0.6 \mathrm{mV}$, at which previous maxima in the differential conductance have turned into minima and vice versa (see also Appendix C).

To emphasize this minimum-maximum transition in the differential conductance, we plot single lines $\Delta G(x)=G(x)-\langle G(x)\rangle$ at equally spaced $V_{\text {sd,dc }}$ between $V_{\mathrm{sd}, \mathrm{dc}}=0 \mathrm{mV}$ and $2 \mathrm{mV}$ in Fig. 2(b). Here, $\langle G(x)\rangle$ is the conductance averaged along $x$ for fixed dc source-drain voltage $V_{\text {sd,dc }}$. At specific fixed tip positions $x$, the differential conductance shows a transition from minima at $\left|V_{\text {sd,dc }}\right|<0.6 \mathrm{mV}$ to maxima at $\left|V_{\text {sd,dc }}\right|>0.6 \mathrm{mV}$. Three of these transitions are marked by the black dashed lines in Fig. 2(b). A similar behavior is also observed for strongly invasive tip potentials, which induce a potential amplitude larger than the Fermi energy in the 2DEG (see Appendix B for measurements at $V_{\text {tip }}=-6 \mathrm{~V}$ ).

Our findings can be put into the context of available theory and the experiment in Ref. [13], in which nonequilibrium carriers are injected through a QPC into an open 2DEG region. In the latter, the observed contrast inversion in regions of branched electron flow at source-drain voltages of up to $V_{\mathrm{sd}, \mathrm{dc}}=2.5 \mathrm{mV}$ is explained based on e-e scattering.
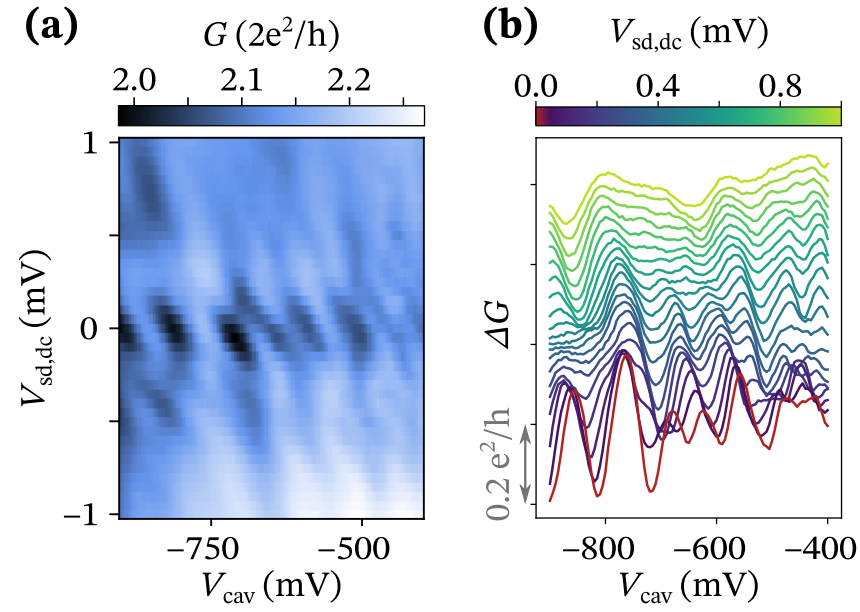

FIG. 3. Differential conductance $G\left(V_{\text {cav }}, V_{\text {sd,dc }}\right)$ in absence of the tip. Corresponding data for source-drain voltages of up to $2 \mathrm{mV}$ can be found in Appendix D. (a) Raw data. (b) $\Delta G\left(V_{\text {cav }}\right)=G\left(V_{\text {cav }}\right)-$ $\left\langle G\left(V_{\text {cav }}\right)\right\rangle$ for small steps in $V_{\text {sd,dc }}$, where $\left\langle G\left(V_{\text {cav }}\right)\right\rangle$ is the average conductance in $V_{\text {cav }}$ at fixed $V_{\text {sd,dc. }}$. The conductance at zero dc bias $\left(V_{\mathrm{sd}, \mathrm{dc}}=0 \mathrm{mV}\right)$ is denoted in red and the lines are offset with respect to each other.

The e-e scattering rate $\tau_{\mathrm{ee}}^{-1}$ depends on the square of the excess energy $\Delta$ above the Fermi energy, at which an electron is injected into the system ( $\tau_{\text {ee }}^{-1} \propto \Delta^{2}$, see discussion below). Increasing the source-drain bias $V_{\text {sd,dc }}$ from zero to $2 \mathrm{mV}$, e-e scattering in the cavity thus becomes particularly relevant for electrons injected at the highest energies.

As reported in Ref. [13], other inelastic scattering mechanisms for hot electrons in 2DEGs (among which the most important ones are plasmon emission [25] and the excitation of acoustic phonons [26]) are irrelevant at the injection energies under investigation (see Appendix A in Ref. [13] for details).

\section{B. Finite bias measurements in absence of the SGM tip}

Signatures of e-e scattering have been shown to be present in many transport experiments in absence of a scanning tip, ranging, e.g., from hydrodynamic flow experiments [27-29] to Young's double-slit [30] and beam splitter [31] experiments. Even though the tip-induced potential in our SGM measurements is smaller than the Fermi energy, it does influence the scattering states in the cavity [cf. Fig. 1(c)]. This raises the question of whether the observed minimummaximum transition is observable only in the presence of the SGM tip or if it is an intrinsic signature of e-e scattering in the cavity also present in the absence of the tip.

In an attempt to resolve this question, we measure the differential conductance $G\left(V_{\text {cav }}, V_{\text {sd,dc }}\right)$ in absence of the tip for a fully formed cavity [ $V_{\text {cav }}$ below the pinch-off voltage]. As the conductance modulations observed in Figs. 1(c) and 2 originate from the local density of states in the cavity, they can be influenced by both the cavity-gate voltage as well as the tip position. The changing cavity gate voltage $V_{\text {cav }}$ thus replaces the varying tip position. Figure 3(a) shows the resulting differential conductance. Again, we observe a clear transition between regions with maximal and minimal conductance, but 
at a slightly different dc voltage of approximately $200 \mu \mathrm{V}$. The shift of the position of the minima and maxima in $V_{\text {cav }}$ arises from the gating of the cavity modes by the bias voltage. This gating effect is even more obvious in Fig. 3(b), which is obtained by the same analysis that was done to obtain Fig. 2(b) (here, the average was taken along $V_{\text {cav }}$ ). Due to this gating effect, it is impossible to identify whether minima in the conductance turn into maxima at higher bias. Figure 3(b) also shows that the shift of the minima/maxima is not linear and therefore cannot be accounted for easily. Therefore, a minimum-to-maximum transition could not be conclusively observed in the absence of the tip.

\section{DISCUSSION}

The e-e scattering length $l_{\mathrm{ee}}$ in the system is given as $l_{\mathrm{ee}}=$ $v_{\mathrm{F}} \tau_{\mathrm{ee}}$, where $v_{\mathrm{F}}$ is the electron velocity at the Fermi energy and $\tau_{\mathrm{ee}}$ is the e-e scattering time. The latter can be estimated to be $[25,28]$

$$
\frac{1}{\tau_{\mathrm{ee}}}=\frac{E_{\mathrm{F}}}{h}\left(\frac{\Delta}{E_{\mathrm{F}}}\right)^{2}\left[\ln \left(\frac{E_{\mathrm{F}}}{\Delta}\right)+\ln \left(\frac{2 q_{\mathrm{TF}}}{k_{\mathrm{F}}}\right)+1\right],
$$

in open two-dimensional electron gases [25] and highmobility quantum wires [28]. Here, $\Delta$ is the excess energy with respect to the Fermi energy $E_{\mathrm{F}}, k_{\mathrm{F}}$ is the Fermi wave vector, and $q_{\mathrm{TF}}$ is the two-dimensional ThomasFermi screening wave vector. Taking the excess energy $\Delta=$ $-|e| V_{\mathrm{sd}, \mathrm{dc}}= \pm 0.6 \mathrm{meV}$ at the minimum-maximum transitions [see Fig. 2(b)], we find an e-e scattering length on the order of $l_{\mathrm{ee}} \approx 3 \mu \mathrm{m}$ in the cavity (cf. Appendix E for further details). This length is on the same order of magnitude as the path length of the round trip between QPC and cavity gate. The lifetime broadening of the cavity modes in the open resonator thus becomes significant with respect to the cavity mode spacing at these injection energies. This may be the reason for the decreasing amplitude of the conductance modulations in Fig. 2(a) with increasing $V_{\text {sd,dc }}$.

Our measurements differ from those in Ref. [13] in two aspects. First, our sample consists of an open resonator formed between a QPC and an arc-shaped cavity gate instead of an open 2DEG behind a QPC. Second, our measurements are obtained with tip-induced potentials lower than $E_{\mathrm{F}}$ instead of the strongly invasive tip potential used in the experiments by Jura et al. [13]. The tip-induced potential in our experiments thus does not backscatter electrons but rather gently deflects their propagation. Surprisingly, despite these differences, our data yield a minimum-to-maximum transition at finite bias voltage, similar to the measurements in Ref. [13].

Because of the complex scattering dynamics in the cavity, the exact microscopic origin of the minimum-to-maximum transition remains elusive. However, the qualitatively similar phenomenology of our data with the results in Ref. [13], as well as the estimates of $l_{\text {ee }}$ given above, suggest the relevance of e-e interactions in the cavity involving the injected nonequilibrium electrons.

\section{CONCLUSIONS}

In conclusion, we measure nonequilibrium transport through an electronic cavity with scanning gate microscopy.
We observe a minimum-to-maximum transition as a function of the source-drain bias $V_{\text {sd,dc }}$ in the differential conductance modulation caused by the tip-induced potential. Our measurements show that gentle electron deflection due to a tip-induced potential below the Fermi energy [17] is sufficient to observe this transition. However, data taken in the absence of the tip show the relevance of the tip-induced potential for the observation of the transition. Despite significant experimental differences, our observations are phenomenologically similar to strongly invasive scanning gate measurements on electrons injected through a point contact into an open two-dimensional electron gas [13]. This suggests a similar microscopic origin of the minimum-to-maximum transition in both experiments, which is based on electron-electron scattering. The detailed microscopic mechanisms of the elaborate scattering processes of electrons in the electronic cavity remain interesting open questions that will require further theoretical and experimental work.

\section{ACKNOWLEDGMENTS}

We thank Leonid Levitov, Vadim Khrapai and Anina Leuch for fruitful discussions and Peter Märki, Thomas Bähler, as well as the staff of the ETH cleanroom facility FIRST for their technical support. We also acknowledge financial support by the ETH Zurich grant ETH-38 17-2 and the Swiss National Science Foundation via NCCR Quantum Science and Technology. M.S. acknowledges support through the National Science Foundation (Grants No. DMR 1709076 and No. ECCS 1508925), and a QuantEmX travel grant from the Institute for Complex Adaptive Matter which is supported by the Gordon and Betty Moore Foundation through Grant No. GBMF5305.

\section{APPENDIX A: INFLUENCE OF THE QPC, CAVITY, AND TIP VOLTAGES ON THE CONDUCTANCE THROUGH THE CAVITY}

To characterize the influence of the QPC and cavity-gate voltages on the conductance through the cavity, we first evaluate the conductance through the QPC in absence of the cavity. The data in Fig. 4(a) shows the conductance quantization characteristic for transport through a quantum point contact. (a)

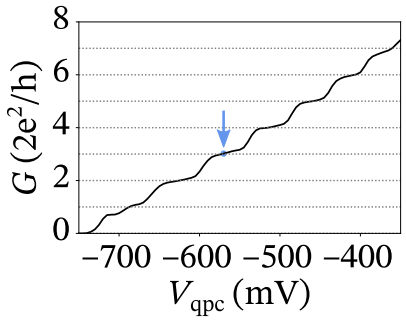

(b)

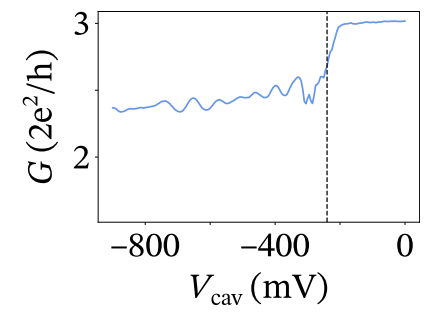

FIG. 4. (a) Conductance quantization of the quantum point contact in absence of the cavity. The blue arrow marks the QPC voltage $V_{\text {qpc }}$ at which the trace in (b) is taken. (b) Conductance through the cavity as a function of the cavity gate voltage for the QPC voltage marked by the blue arrow in (a) (third conductance plateau). The dashed line marks the pinch-off voltage of the cavity gate. 
Setting the QPC to the third conductance plateau [cf. the blue arrow in Fig. 4(a)], we then study the influence of the cavity-gate voltage on the conductance through the sample. The data in Fig. 4(b) shows that small cavity-gate voltages do not influence the conductance significantly. Thus, the conductance remains similar to the conductance plateau in absence of the cavity for cavity-gate voltages more positive than the pinch-off voltage [cf. the black dashed line in Fig. 4(b)]. For cavity-gate voltages more negative than the pinch-off voltage, the cavity gate depletes the 2DEG below the gate and the cavity forms. As the capacitive coupling between the cavity gate and the QPC reduces the conductance through the QPC, we observe a reduced conductance of $G \approx 2.4 \cdot 2 e^{2} / h$ through the fully formed cavity. The emergence of quantized modes within the cavity furthermore yields the characteristic conductance modulations observed in Fig. 4(b). Despite the influence of the cavity gate on the QPC, the QPC still features welldefined conductance plateaus in the presence of the cavity [cf. Fig. 1(b)].

While imaging the cavity with a SGM tip, the capacitive coupling between the tip and the QPC further reduces the conductance through the cavity. Commonly, the tip-induced potential is described by a Lorentzian shaped potential [32-35]. Thus, the tip couples strongest to the QPC for tip positions in close vicinity of the QPC [cf. Fig. 1(c)]. However, the long-ranged tails of the tip potential have a small, decreasing [for increasing tip-QPC distances], yet nonnegligible influence on the QPC. The combined action of the capacitive coupling of the cavity and tip with the QPC thus reduces the average conductance in Fig. 1(c) with respect to the conductance $G=3 \cdot 2 e^{2} / h$ in absence of both the tip and cavity potential.

\section{APPENDIX B: FINITE-BIAS MEASUREMENTS WITH STRONGLY INVASIVE TIP POTENTIALS}

We evaluate the influence of a strongly invasive tip potential on the observation of the minimum-to-maximum transitions by repeating the measurements depicted in Fig. 2 for $V_{\text {tip }}=-6 \mathrm{~V}$. The data is depicted in Fig. 5. In accordance with previous experiments [17], the additional scattering of electrons off the strongly invasive tip potential results in sharper and denser conductance modulations in the cavity area. Nonetheless, Fig. 5 exhibits the same minima-to-maxima transition observed in Fig. 2. Therefore, the observation of the minima-to-maxima transitions is independent of the strength of the tip-induced potential.

\section{APPENDIX C: FINITE-BIAS MEASUREMENTS WITH WEAKLY INVASIVE TIP POTENTIALS}

To further highlight the minima-to-maxima transition observed in Fig. 2, we calculate the conductance difference $\Delta G\left(x, V_{\mathrm{sd}, \mathrm{dc}}\right)$ as discussed in Sec. IV A. The resulting data, depicted in Fig. 6, features transitions from minima (blue) to maxima (red).
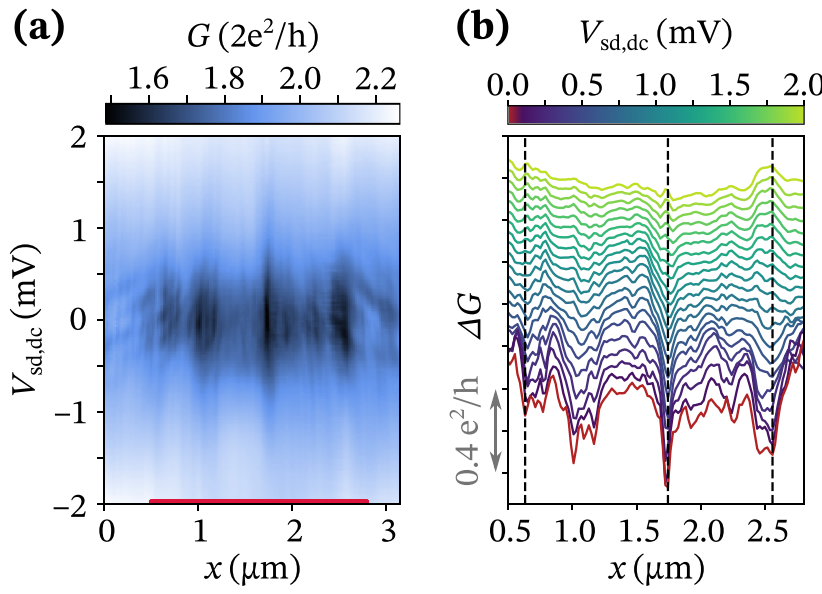

FIG. 5. Differential conductance $G\left(x, V_{\text {sd,dc }}\right)$ for a strongly invasive tip potential $\left(V_{\text {tip }}=-6 \mathrm{~V}\right)$ along a similar line as the red dashed line in Fig. 1(c). (a) Raw data. (b) Conductance difference $\Delta G(x)$ for tip positions $x$ along the red line in (a) and small steps in $V_{\mathrm{sd}, \mathrm{dc}}$. The red curve corresponds to zero source-drain bias and the lines are offset with respect to each other. Black dashed guides to the eye denote three exemplary minimum-to-maximum transitions.

\section{APPENDIX D: FINITE-BIAS MEASUREMENTS IN ABSENCE OF THE TIP: FULL DATA RANGE}

The behavior of the conductance modulations for sourcedrain voltages beyond $V_{\mathrm{sd} \text {,dc }}=1 \mathrm{mV}$ in absence of the tip (cf. Fig. 3) is depicted in Fig. 7. For higher source-drain voltages, we observe a rather stable evolution of the conductance without any further minima-to-maxima transitions.

\section{APPENDIX E: $V_{\text {sd,dc }}$-DEPENDENCE OF THE CAVITY MODULATIONS IN PRESENCE OF THE TIP}

The estimate of the e-e scattering time [cf. Eq. (1)] in Sec. V is reported for electron transport in open twodimensional electron gases [25] and high-mobility quantum wires [28]. As the number of available states determines the prevalence of e-e scattering events, the reduced density of states in the open resonator possibly reduces the number of

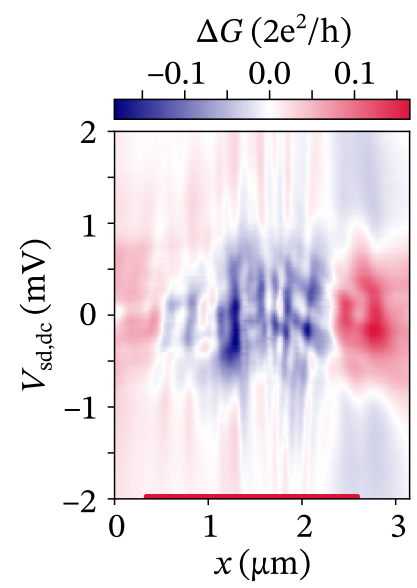

FIG. 6. Conductance difference $\Delta G\left(x, V_{\mathrm{sd}, \mathrm{dc}}\right)$ of the data depicted in Fig. 2(a). 

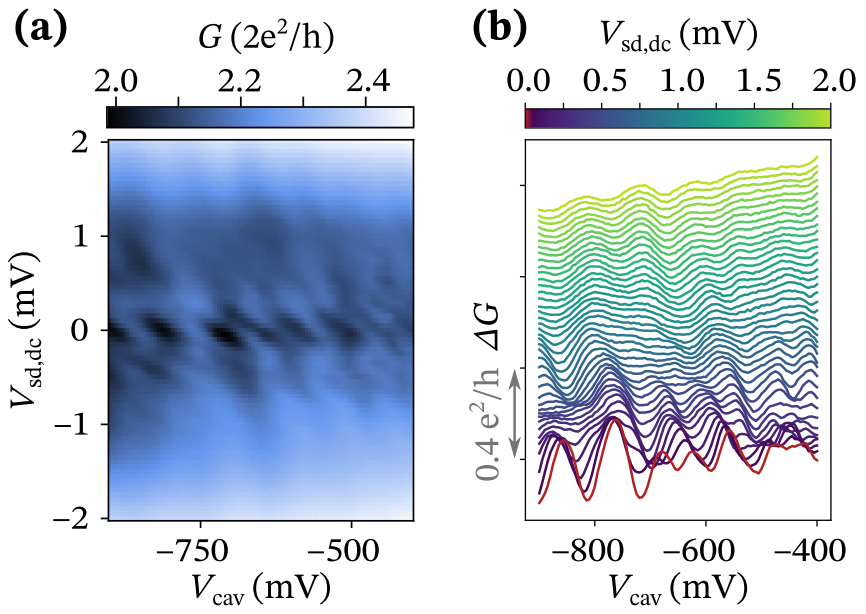

FIG. 7. Differential conductance $G\left(V_{\text {cav }}, V_{\text {sd,dc }}\right)$ depicted in Fig. 3 for a larger range of $V_{\mathrm{sd}, \mathrm{dc}}$.

e-e interactions in the latter. To evaluate the influence of the reduced number of states available for scattering in the cavity on the e-e scattering events, we revisit the data depicted in Fig. 2. Figure 8 shows the conductance difference $\Delta G\left(V_{\mathrm{sd}, \mathrm{dc}}\right)$ for small steps in the tip position $x$ along the red line in Fig. 1(c). At low bias voltages, the conductance modulations inside the cavity make it impossible to observe a distinct behavior of the conductance as a function of the source-drain bias. In contrast to this, we observe a parabolic dependence of the conductance

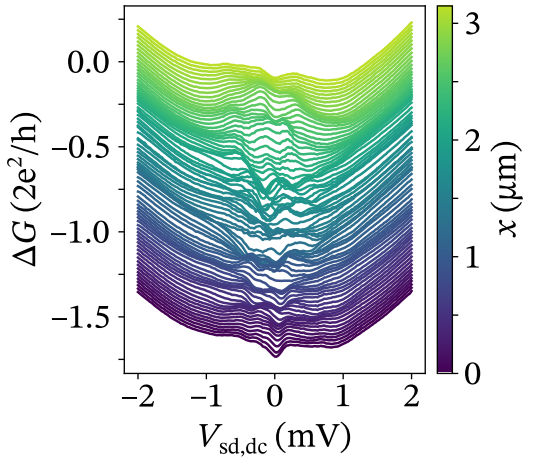

FIG. 8. Conductance difference $\Delta G\left(V_{\mathrm{sd}, \mathrm{dc}}\right)$ for small steps in the tip positions $x$ along the red line in Fig. 2(a).

difference $\Delta G\left(V_{\mathrm{sd}, \mathrm{dc}}\right)$ on the source-drain bias at high bias voltages. This parabolic dependence is in good agreement with the dependence of the e-e scattering rate on the excess energy $\Delta=-|e| V_{\text {sd,dc }}$ [cf. Eq. (1), Refs. [25,28]]. In particular, we observe that the dependence of $\Delta G$ on the source-drain bias $V_{\mathrm{sd}, \mathrm{dc}}$ is uniform for all tip positions across the cavity and does not vary for tip positions outside ( $x \approx 0 \mu \mathrm{m}, x \approx 3 \mu \mathrm{m})$ and inside the cavity. This suggests that the cavity is still embedded in the Fermi sea, and thus provides sufficient available states for e-e scattering. While this observation suggests the qualitative validity of Eq. (1), it does not allow conclusions about the quantitative accuracy of Eq. (1) for e-e scattering in the cavity.
[1] K. Seeger, Semiconductor Physics An Introduction, 5th ed., Springer Series in Solid-State Sciences, Vol. 40 (Springer Berlin Heidelberg, Berlin, Heidelberg, 1991).

[2] A. L. Ėfros and M. Pollak, eds., Electron-Electron Interactions in Disordered Systems, Modern Problems in Condensed Matter Sciences, Vol. 10 (North-Holland, Amsterdam, 1985).

[3] G. Zala, B. N. Narozhny, and I. L. Aleiner, Interaction corrections at intermediate temperatures: Longitudinal conductivity and kinetic equation, Phys. Rev. B 64, 214204 (2001).

[4] Y. Imry, Introduction to Mesoscopic Physics, 2nd ed., Mesoscopic Physics and Nanotechnology (University Press, Oxford, 2009).

[5] Y. Aharonov and D. Bohm, Significance of electromagnetic potentials in the quantum theory, Phys. Rev. 115, 485 (1959).

[6] R. A. Webb, S. Washburn, C. P. Umbach, and R. B. Laibowitz, Observation of $h / e$ Aharonov-Bohm Oscillations in NormalMetal Rings, Phys. Rev. Lett. 54, 2696 (1985).

[7] A. E. Hansen, A. Kristensen, S. Pedersen, C. B. Sørensen, and P. E. Lindelof, Mesoscopic decoherence in Aharonov-Bohm rings, Phys. Rev. B 64, 045327 (2001).

[8] G. Bergmann, Weak localization in thin films: A time-offlight experiment with conduction electrons, Phys. Rep. 107, 1 (1984).

[9] D. A. Bandurin, I. Torre, R. K. Kumar, M. B. Shalom, A. Tomadin, A. Principi, G. H. Auton, E. Khestanova, K. S. Novoselov, I. V. Grigorieva, L. A. Ponomarenko, A. K. Geim, and M. Polini, Negative local resistance caused by viscous electron backflow in graphene, Science 351, 1055 (2016).
[10] B. A. Braem, F. M. D. Pellegrino, A. Principi, M. Röösli, C. Gold, S. Hennel, J. V. Koski, M. Berl, W. Dietsche, W. Wegscheider, M. Polini, T. Ihn, and K. Ensslin, Scanning gate microscopy in a viscous electron fluid, Phys. Rev. B 98 , 241304(R) (2018).

[11] D. M. Zumbühl, C. M. Marcus, M. P. Hanson, and A. C. Gossard, Asymmetry of Nonlinear Transport and Electron Interactions in Quantum Dots, Phys. Rev. Lett. 96, 206802 (2006).

[12] D. Taubert, C. Tomaras, G. J. Schinner, H. P. Tranitz, W. Wegscheider, S. Kehrein, and S. Ludwig, Relaxation of hot electrons in a degenerate two-dimensional electron system: Transition to one-dimensional scattering, Phys. Rev. B 83, 235404 (2011).

[13] M. P. Jura, M. Grobis, M. A. Topinka, L. N. Pfeiffer, K. W. West, and D. Goldhaber-Gordon, Spatially probed electronelectron scattering in a two-dimensional electron gas, Phys. Rev. B 82, 155328 (2010).

[14] M. A. Topinka, B. J. LeRoy, S. E. J. Shaw, E. J. Heller, R. M. Westervelt, K. D. Maranowski, and A. C. Gossard, Imaging coherent electron flow from a quantum point contact, Science 289, 2323 (2000).

[15] M. A. Topinka, B. J. LeRoy, R. M. Westervelt, S. E. J. Shaw, R. Fleischmann, E. J. Heller, K. D. Maranowski, and A. C. Gossard, Coherent branched flow in a two-dimensional electron gas, Nature 410, 183 (2001).

[16] M. P. Jura, M. A. Topinka, L. Urban, A. Yazdani, H. Shtrikman, L. N. Pfeiffer, K. W. West, and D. Goldhaber-Gordon, Un- 
expected features of branched flow through high-mobility two-dimensional electron gases, Nat. Phys. 3, 841 (2007).

[17] R. Steinacher, C. Pöltl, T. Krähenmann, A. Hofmann, C. Reichl, W. Zwerger, W. Wegscheider, R. A. Jalabert, K. Ensslin, D. Weinmann, and T. Ihn, Scanning gate experiments: From strongly to weakly invasive probes, Phys. Rev. B 98, 075426 (2018).

[18] C. Yan, S. Kumar, M. Pepper, P. See, I. Farrer, D. Ritchie, J. Griffiths, and G. Jones, Interference Effects in a Tunable Quantum Point Contact Integrated with an Electronic Cavity, Phys. Rev. Appl. 8, 024009 (2017).

[19] C. Yan, S. Kumar, M. Pepper, P. See, I. Farrer, D. Ritchie, J. Griffiths, and G. Jones, Incipient singlet-triplet states in a hybrid mesoscopic system, Phys. Rev. B 97, 241302(R) (2018).

[20] C. Yan, S. Kumar, P. See, I. Farrer, D. Ritchie, J. P. Griffiths, G. A. C. Jones, and M. Pepper, Magnetoresistance in an electronic cavity coupled to one-dimensional systems, Appl. Phys. Lett. 113, 112101 (2018).

[21] C. Gold, B. A. Bräm, M. S. Ferguson, T. Krähenmann, A. Hofmann, R. Steinacher, K. R. Fratus, C. Reichl, W. Wegscheider, D. Weinmann, K. Ensslin, and T. Ihn, Imaging signatures of the local density of states in an electronic cavity, arXiv:2011.13869.

[22] M. A. Eriksson, R. G. Beck, M. A. Topinka, J. A. Katine, R. M. Westervelt, K. L. Campman, and A. C. Gossard, Effect of a charged scanned probe microscope tip on a subsurface electron gas, Superlattices Microstruct. 20, 435 (1996).

[23] J. A. Katine, M. A. Eriksson, A. S. Adourian, R. M. Westervelt, J. D. Edwards, A. Lupu-Sax, E. J. Heller, K. L. Campman, and A. C. Gossard, Point Contact Conductance of an Open Resonator, Phys. Rev. Lett. 79, 4806 (1997).

[24] C. Rössler, D. Oehri, O. Zilberberg, G. Blatter, M. Karalic, J. Pijnenburg, A. Hofmann, T. Ihn, K. Ensslin, C. Reichl, and W. Wegscheider, Transport Spectroscopy of a SpinCoherent Dot-Cavity System, Phys. Rev. Lett. 115, 166603 (2015).
[25] G. F. Giuliani and J. J. Quinn, Lifetime of a quasiparticle in a two-dimensional electron gas, Phys. Rev. B 26, 4421 (1982).

[26] T. Ihn, Semiconductor Nanostructures: Quantum States and Electronic Transport (Oxford University Press, Oxford, New York, 2009).

[27] L. W. Molenkamp and M. J. M. de Jong, Observation of Knudsen and Gurzhi transport regimes in a two-dimensional wire, Solid-State Electron. 37, 551 (1994).

[28] M. J. M. de Jong and L. W. Molenkamp, Hydrodynamic electron flow in high-mobility wires, Phys. Rev. B 51, 13389 (1995).

[29] G. M. Gusev, A. D. Levin, E. V. Levinson, and A. K. Bakarov, Viscous electron flow in mesoscopic two-dimensional electron gas, AIP Adv. 8, 025318 (2018).

[30] A. Yacoby, U. Sivan, C. P. Umbach, and J. M. Hong, Interference and Dephasing by Electron-Electron Interaction on Length Scales Shorter than the Elastic Mean Free Path, Phys. Rev. Lett. 66, 1938 (1991).

[31] X. Ying, J. P. Lu, J. J. Heremans, M. B. Santos, M. Shayegan, S. A. Lyon, M. Littman, P. Gross, and H. Rabitz, Quantum reflection and transmission of ballistic two-dimensional electrons by a potential barrier, Appl. Phys. Lett. 65, 1154 (1994).

[32] M. A. Eriksson, R. G. Beck, M. Topinka, J. A. Katine, R. M. Westervelt, K. L. Campman, and A. C. Gossard, Cryogenic scanning probe characterization of semiconductor nanostructures, Appl. Phys. Lett. 69, 671 (1996).

[33] M. G. Pala, B. Hackens, F. Martins, H. Sellier, V. Bayot, S. Huant, and T. Ouisse, Local density of states in mesoscopic samples from scanning gate microscopy, Phys. Rev. B 77, 125310 (2008).

[34] R. Steinacher, A. A. Kozikov, C. Rössler, C. Reichl, W. Wegscheider, T. Ihn, and K. Ensslin, Scanning-gate-induced effects and spatial mapping of a cavity, New J. Phys. 17, 043043 (2015).

[35] B. Brun, N. Moreau, S. Somanchi, V.-H. Nguyen, K. Watanabe, T. Taniguchi, J.-C. Charlier, C. Stampfer, and B. Hackens, Imaging Dirac fermions flow through a circular Veselago lens, Phys. Rev. B 100, 041401(R) (2019). 